\title{
The Influence of Fixed and Moving NPC on Pedestrians' Avoidance Behaviors: VR-Based Experiments
}

\author{
Weisong Liu · Jun Zhang · Weiguo Song \\ University of Science and Technology of China, Hefei, China \\ E-mail: Iws2030@mail.ustc.edu.cn; junz@ustc.edu.cn, wgsong@ustc.edu.cn
}

Received: 13 August 2021 / Last revision received: 10 November 2021 / Accepted:11 November 2021

DOI: $10.17815 / C D .2021 .122$

\begin{abstract}
Pedestrians have to take actions to avoid collisions with other objects. Pedestrians take different avoidance measures to avoid standing object and moving standing. Finding the difference between pedestrian avoidance in the two situations will helps to establish the avoidance model. In this work, we focus on the differences of avoidance behaviors when a pedestrian meets a moving or fixed intruder (Non-player character) in a virtual environment. In moving NPC experiments, the distance between start avoidance point and the potential collision point (CP) first decreases and then increases with the increasing intrusion angle of the intruder. In standing NPC experiments, pedestrians start to avoid closer to the CP. In moving NPC experiments, the average maximum lateral offset distance (MLD) for the pedestrians to detour decreases with the decreasing intrusion angles of the intruder. In standing NPC experiments, the average MLD is $1.01 \mathrm{~m}$. What's more, at the intrusion angles of $30^{\circ}, 60^{\circ}, 90^{\circ}$ and $120^{\circ}$, the pedestrians detouring behind the NPC require larger MLD than those detouring in front of the NPC. Thus, more subjects prefer to avoid potential conflicts by detouring in front of the NPC under these conditions. But the preference weakens and disappears at $150^{\circ}$ and $180^{\circ}$ intrusion angles due to the decreasing of MLD. In standing NPC experiments, such tendency is not observed in pedestrians' avoidance strategies (right: $46 \%$, left: $54 \%$ ). This article quantitatively analyses the difference between the influence of fixed and moving NPC on pedestrians' avoidance strategies. The mechanism of pedestrian's avoidance behavior is obtained by analyzing characteristic parameters, which is helpful to adjust pedestrian avoidance prediction models and design humanoid robots.
\end{abstract}

Keywords Pedestrians' avoidance behavior · virtual reality $\cdot$ moving NPC $\cdot$ fixed NPC 


\section{Introduction}

In daily life, we will encounter pedestrians in two states, standing or moving. Pedestrians in different states have different influence on the movement of others. For example, a pedestrian (obstacle) standing on another person's route and a pedestrian moving to the same position on the route may have different effects on others. Pedestrians will take different avoidance measures in different situations. There are few studies on this difference at present. Thus, two experiments (Moving NPC experiment and Standing NPC experiment) are conducted to study the differences between the influence of standing NPC and moving NPC on pedestrians' avoidance behaviors. NPC represents the character that is not manipulated by real players. This research contributes to the development of robot's avoidance models.

In recent years, some scholars have conducted relevant research on the pedestrians' avoidance strategies. In the previous research on static obstacles (pedestrian), a series of experiments have been conducted. Lv et al. [1] propose the reverse cumulative probability to represent the probability of the pedestrian turning in front of single fixed obstacle. Bourgaize et al. [2] find that if the obstacle has larger size, the lateral offset of the pedestrians will increase slightly. Pedestrians take avoiding measures at an average distance of $5.06 \mathrm{~m}$ from the fixed obstacle. In the previous research on movement obstacles (pedestrian), a series of experiments have been conducted. Knorr et al. [3] study the influence of person-specific and situation-specific characteristics on collision avoidance behavior in human locomotion. They find that the pedestrians' avoidance behavior is mainly affected by situation-specific characteristics, such as walking speed and heading direction rather than gender, height. Lappe et al. [4] believe that the pedestrian avoidance strategy is related to the crossing angle and walking speed of both parties. Therefore, the pedestrians' avoidance behavior may be different when facing different types of obstacles.

By comparing the results of previous studies, it can be found that there are significant differences in the avoidance distances of pedestrians when facing different obstacles. Lv et al. find that most pedestrians would change direction when they are 0.9-2.0 m away from the fixed obstacle [1]. Parisi found that, when one pedestrian is arrested and not moving, the minimum distance of encounter between two pedestrians is approximately $0.75 \mathrm{~m}$. But when both pedestrians are moving, the minimum avoidance distance is approximately $1 \mathrm{~m}$ in the case of a potential perpendicular collision [5]. Besides, it has been revealed that the average critical walking space was about $2 \mathrm{~m}^{2}$ for avoiding a standing person and $2.64 \mathrm{~m}^{2}$ for avoiding an opposite moving pedestrian [6]. In other conditions when avoiding a moving pedestrian, the variation of crossing angle would also influence the avoiding distance [7]. The above research results indicate that different obstacles have different impact ranges on pedestrians. Therefore, pedestrians will take different avoidance strategies when facing fixed obstacle and moving obstacle. At present, the research about this topic is relatively scarce.

In recent years, virtual reality (VR) has become a behavior analysis tool [8]. E.g. Kinateder et al. [9] conducted an evacuation experiment in a virtual library. The study found that participants prefer to evacuate from familiar exits. Participants' exit choice is affected by the surrounding pedestrians, and this influence will increase as the number 
of surrounding pedestrians increases. Baxter et al. [10] study how fixed and extended obstacles with different positions and rotation angles affect the pedestrian's avoidance behavior. They find that when the obstacle rotation angles are $45^{\circ}$ and $135^{\circ}$, pedestrians have a significant preference of avoiding from left or right side. Bruneau et al. [11] study on individual avoidance of groups (Either walker go through or around the group) using VR. They find that when the group interpersonal distance is $1.7 \mathrm{~m}$, there is no preference for the two strategies. Sean et al. [12] find that the final crossing distance is affected by the global motion appearances (the shape of obstacle). Unity 3D is a tool for creating interactive 3D applications on multiple platforms. Unity3D consists of a game engine and an Editor. The engine contains software components for the most common and recurring tasks in game development. The topics covered by the engine include sound, graphics, physics and network functions. The physics engine in Unity can accurately set fixed dynamic parameters of pedestrians, so as to solve the problem of constantly changing dynamic parameters of participants in reality, which is helpful for in-depth analyses of the pedestrians' avoidance behavior.

The differences between standing NPC and moving NPC are observed in this study based on VR technique. The rest of the paper is structured as follows. Sec. 2 gives the experimental setup, procedure, etc. of the experiments. The results about pedestrian's avoidance behaviors are presented in Sec. 3. Sec. 4 discusses the results of this article and future work.

\section{Methods}

\subsection{Experimental setup}

Unity 3D was used to build a virtual street scene (Length $\times$ Width: $40 \mathrm{~m} \times 40 \mathrm{~m}$ ) to investigate pedestrians' avoidance behaviors (Fig. 1). HTC VIVE VR system connecting with computer workstation was used for visual data output. Participants need to avoid the moving NPC or standing NPC on the street. Initially, each participant was located at the position $(0,0)$, who was supposed to walk to the target $(0,30)$. The first perspective of the experimental scene is shown in Fig. 2. In the moving NPC experiment, an invisible circle with the radius of $10 \mathrm{~m}$ and the center of $(0,15)$ was used to determine the initial positions of the NPC in the experiment, which made sure that the NPC and pedestrians would collide at collision point $(0,15)$ when non avoidance measures were taken. Subject and NPC had the same speed of $1.5 \mathrm{~m} / \mathrm{s}$. In the standing NPC experiment, NPC was fixed at the collision point (CP). Pedestrians need to walk at $1.5 \mathrm{~m} / \mathrm{s}$ to the same target. In both experiments, we set up capsule colliders (Diameter: $0.5 \mathrm{~m}$, Height: $1.7 \mathrm{~m}$ ) on the agent of subject and the NPC. If the subjects didn't take any avoidance measures, they would collide with NPC at the CP.

In the moving NPC experiments, two independent variables to investigate the influence of moving NPC on the avoidance movement: NPC's intrusion angle, NPC's intrusion side. (1) NPC's intrusion angle (6 levels): The angle between the NPC's movement route and the subjects' desired route (the white dotted line), including $30^{\circ}, 60^{\circ}, 90^{\circ}, 120^{\circ}, 150^{\circ}$ 
and $180^{\circ}$. (2) NPC's intrusion side (2 levels): the NPC moved to the Center of the circle from the left or right side of the subjects (Fig. 1). This created a 2-way mixed design for statistical analyses, which yielding 12 experimental conditions.

\begin{tabular}{ccccccccc|cccccc}
\hline Category & Variable & \multicolumn{1}{c}{ Value } \\
\hline \multirow{2}{*}{ Moving NPC } & Side & \multicolumn{1}{c}{ Left } & \multicolumn{1}{c}{ Right } \\
& Angle & 30 & 60 & 90 & 120 & 150 & 180 & 30 & 60 & 90 & 120 & 150 & 180 \\
\hline
\end{tabular}

Table 1 The setup of the independent variables

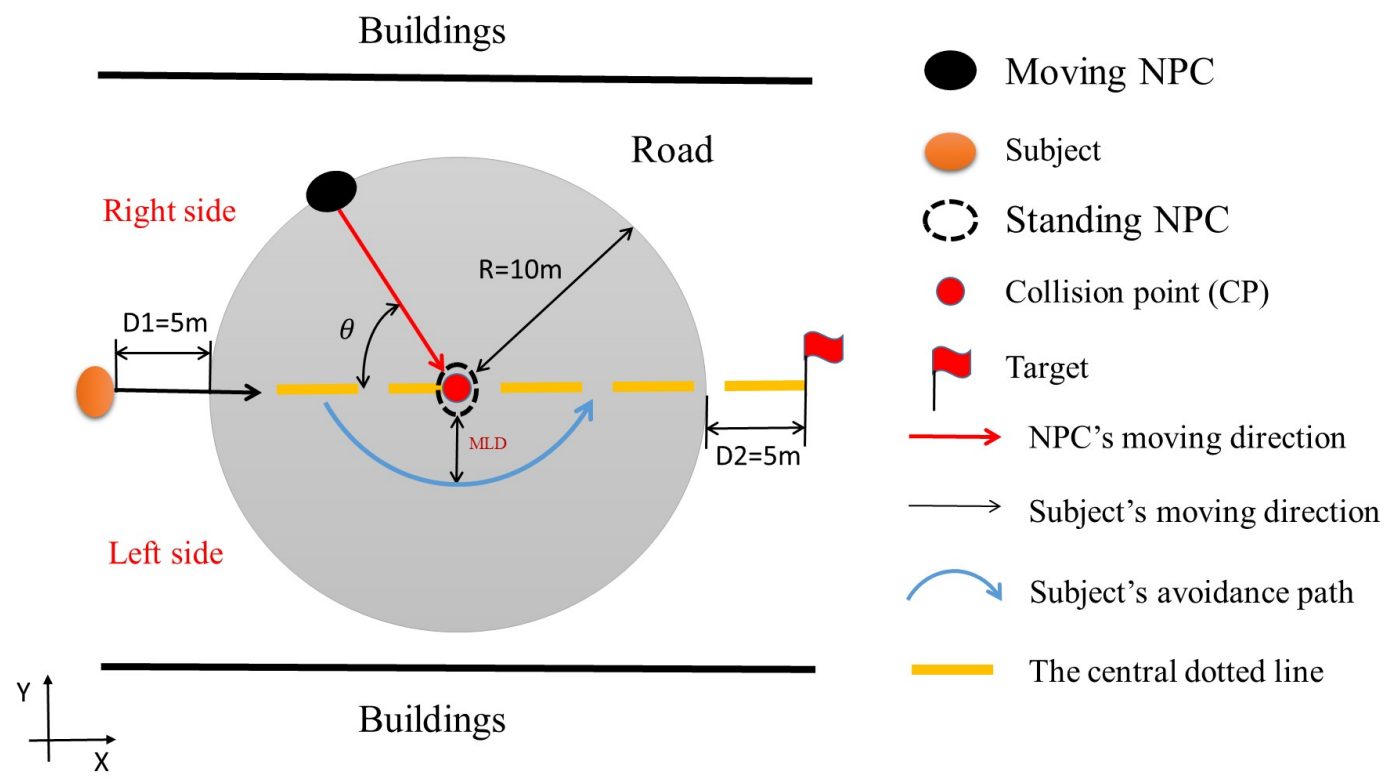

Figure 1 The concept map of experimental scene

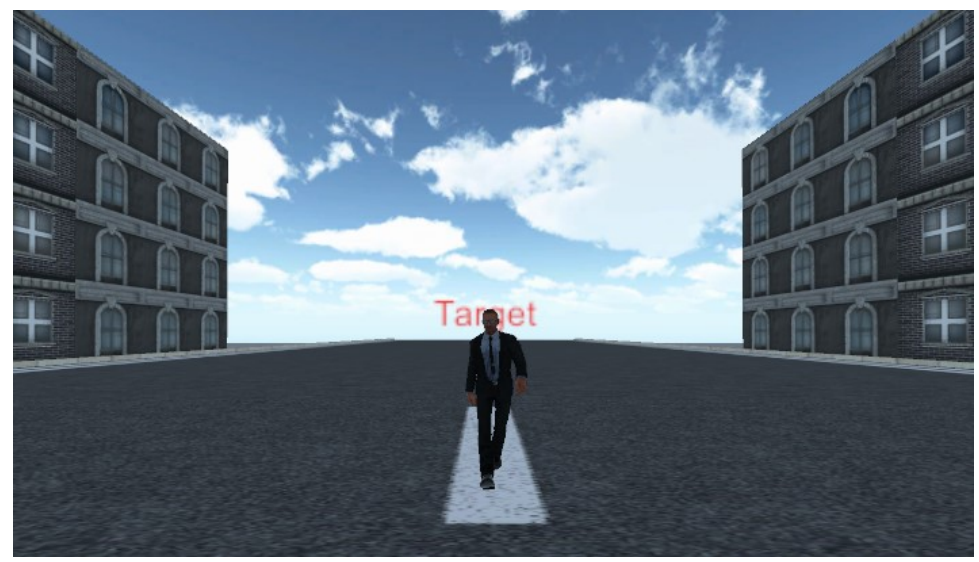

Figure 2 The first perspective of the experimental scene 


\subsection{Participants}

The participants were recruited subjects from campus and not supposed to have any heartrelated illness, head diseases and severe 3DVertigo. In standing NPC experiments, 37 (21 males and 16 females) subjects are recruited to finished 37 sets of experiments. In moving NPC experiments, 42 participants (22 males and 20 females) finished 252 sets of experiments. The ages of participants ranged from 16 to 33 with an average of 23.1 years old. The one who wears eyeglasses or contact lenses in their daily life continued to wear them in the experiment. All of them completed the experiment with slight or no discomfort. They received a souvenir as a reward for participation in this study.

\subsection{Procedure}

This study was approved by the University of Science and Technology of China. At the beginning of the experiment, the health conditions of the participants were asked. Only the suitable candidates were adopted to continue for the following procedures. Meanwhile, the participants were informed that they could quit the experiment at any time if they felt uncomfortable.

Next was the training trial, which was the same as the formal experiment, but without NPC. Participants were asked to wear the headsets and kept their position at the calibration center point. During the training experiments, they were taught how to navigate in the VE. The subjects walked in the scene via pressing the 'w' key, which should be kept holding down during the experiment. There was an experimenter who could provide help if the participants met any technical problems. When they were familiar with the experiment operations, the formal experiments would be conducted.

At the beginning of the experiment, the participants were told to walk along the central white line to the target as much as possible. However, if the subjects' movement was affected by NPC (they think they may collide with NPC), they could take avoidance measures (deviate from the white line). In the moving NPC experiment, once a participant arrived at the invisible circle $(0,5)$, the NPC with an intrusion angle of $\theta$ started to move to the center of the circle at a fixed speed of $1.5 \mathrm{~m} / \mathrm{s}$. In standing NPC experiment, the NPC maintained a standing posture at the CP and faced toward the subject's initial position. When the pedestrian moved to the target along the central white dotted line, one experiment ended. It should be noted that the direction of subject toward the target point (desired direction) was the $\mathrm{X}$ axis positive direction (The vertical axis in Fig. 1). During an experiment, if the physics engine detects a collision between a pedestrian and NPC, the experiment will automatically stop and restart.

\section{Experiment results}

\subsection{Pedestrians' trajectories}

The pedestrians' trajectories in moving NPC (only right side) and standing NPC experiments are shown in Fig. 3 and Fig. 4. The positive direction of the X coordinates is the 
desired moving direction of pedestrians. The Y coordinates represents the lateral offset distance.

The pedestrians' avoidance process can be divided into three stages: Judgment stage - Avoidance stage - Return stage. In the judgment stage, participants observe the locomotion of the NPC. When they decide to start avoiding the NPC, it enters the second stage. The return stage starts when the participants reach the maximum lateral deviation (MLD). In standing NPC experiments, the shapes of trajectories are left-right symmetry. But in moving NPC experiments, the shapes of trajectories are asymmetric, especially at $30^{\circ}, 60^{\circ}, 90^{\circ}$ intrusion angles. In the following few sections, the characteristics of start avoidance point and MLD will be analyzed. 


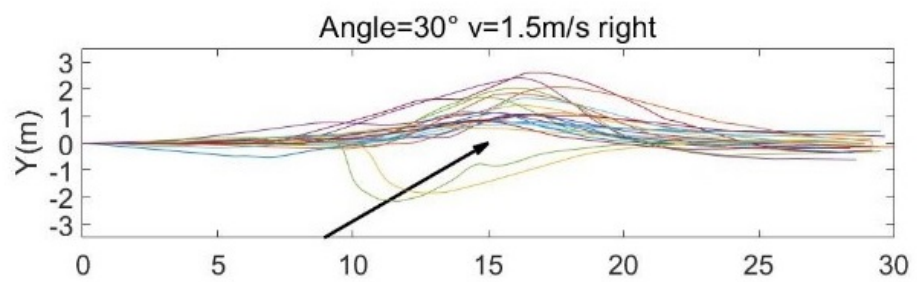

Angle $=60^{\circ} \mathrm{v}=1.5 \mathrm{~m} / \mathrm{s}$ right

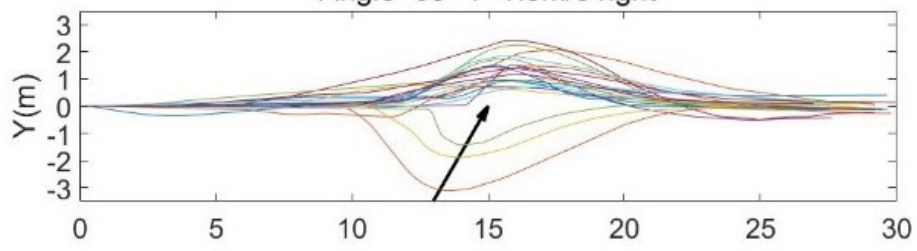

Angle $=90^{\circ} \mathrm{v}=1.5 \mathrm{~m} / \mathrm{s}$ right

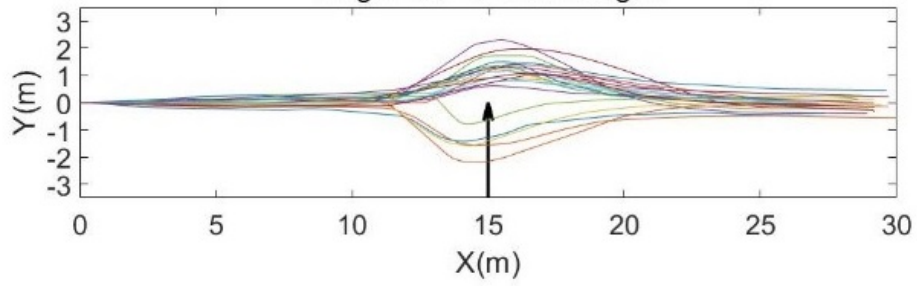

(a)

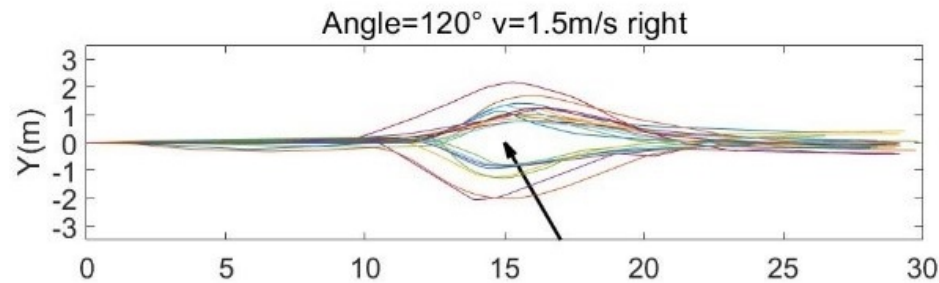

Angle $=150^{\circ} \mathrm{v}=1.5 \mathrm{~m} / \mathrm{s}$ right

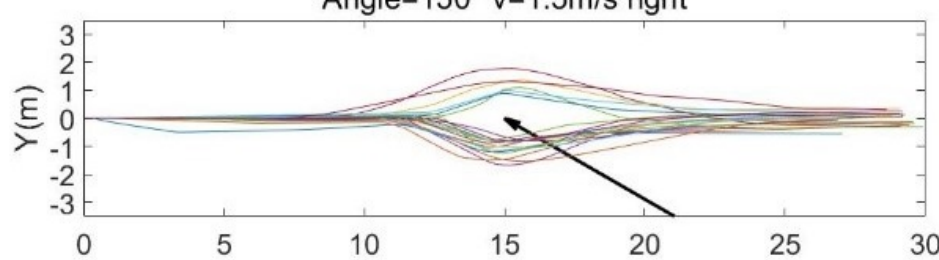

Angle $=180^{\circ} \mathrm{v}=1.5 \mathrm{~m} / \mathrm{s}$ right

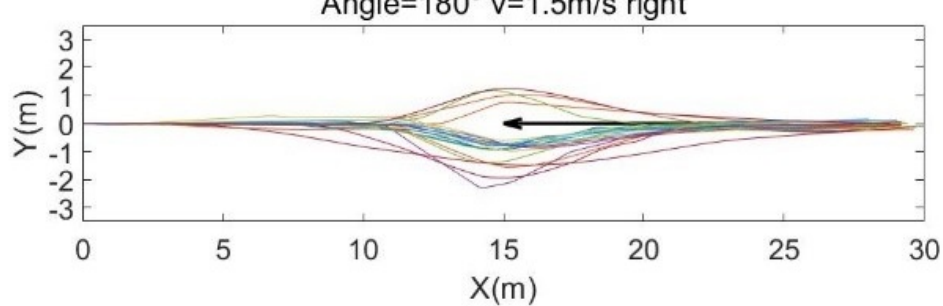

(b)

Figure 3 The pedestrians' trajectories (NPC comes from right side). The trajectories of NPC are shown as the black line with arrow. 


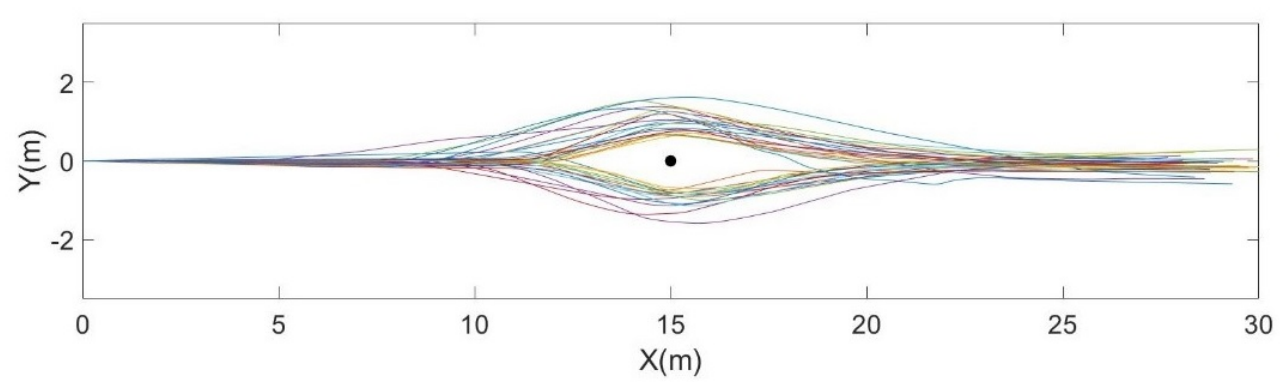

Figure 4 The pedestrians' trajectories (standing NPC). The point represents the standing NPC.

\subsection{Avoidance probability distribution and Preference range}

The position where participant need to take avoidance measures is defined as the starting avoidance point. In this work, the starting avoidance point is defined as the pedestrian's moving direction deviates from the $\mathrm{X}$-axis by more than $5^{\circ}[13]$ and the lateral deviation from the $\mathrm{X}$-axis is greater than $10 \mathrm{~cm}$. In order to study the distribution of the start avoidance point, the $10 \mathrm{~m}$ area in front of the CP is divided into 10 zones ( $1 \mathrm{~m}$ per zone) along the X-axis (Fig. 5).

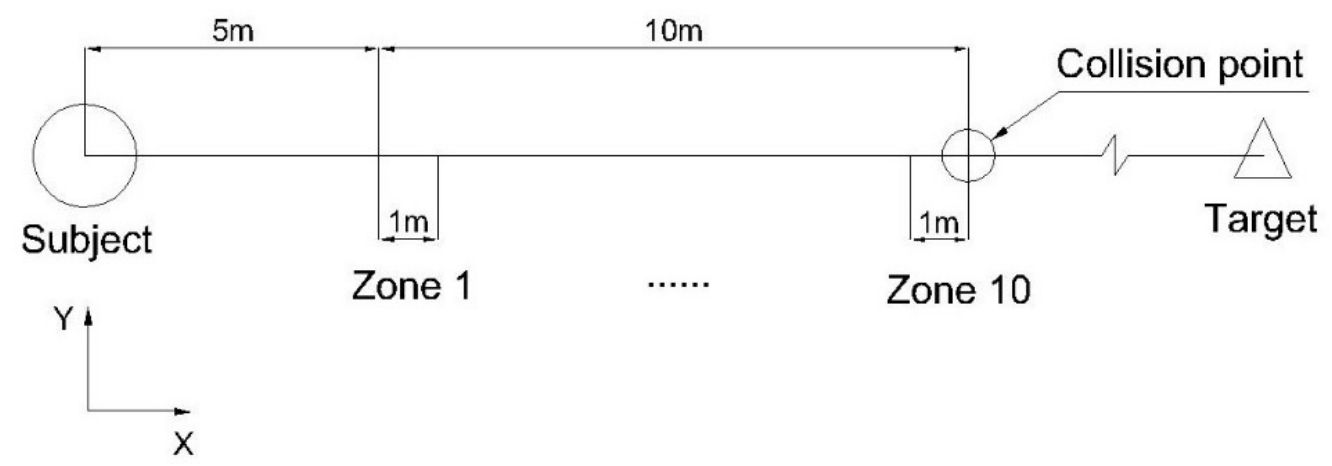

Figure 5 The division of the 10 zones before the CP

The average distances from start avoidance point to $\mathrm{CP}$ along the $\mathrm{X}$-axis are studied and listed in Tab. 2. It's found that the distance between the start avoidance point and $\mathrm{CP}$ first decreases and then increases with the increasing intrusion angle in moving NPC experiments. In standing NPC experiments, the start avoidance points are closer $(3.73 \mathrm{~m}$ in average) to $\mathrm{CP}$.

Compared with a moving NPC, the pedestrian will take avoidance measures later when facing a standing NPC. When facing a moving NPC, pedestrians need to predict the future movement position of the NPC. Due to the ambiguity and inaccuracy of the prediction, pedestrians start avoiding early. But in standing NPC experiments, there is no need to 


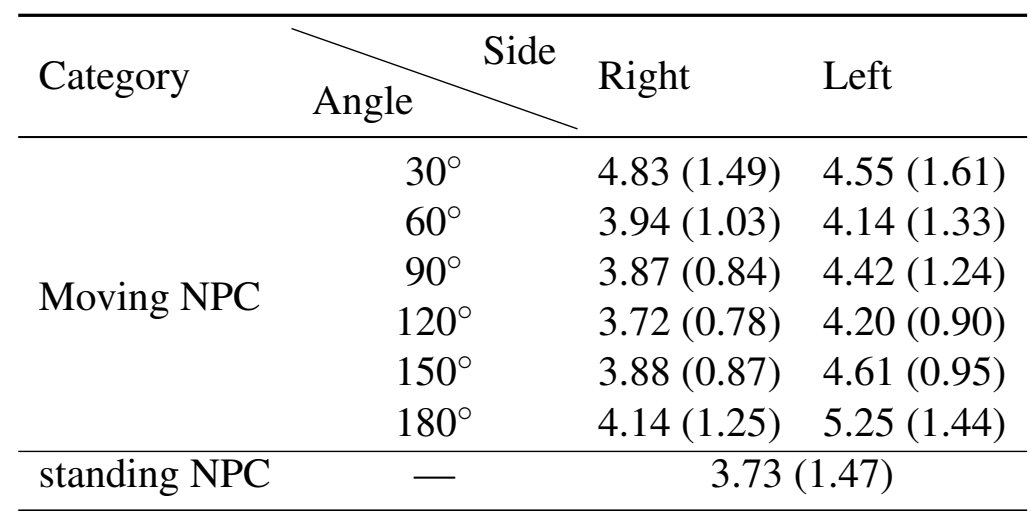

Table 2 The average location of pedestrians' start avoidance points (Unit:m). The standard deviation of the data is shown in parentheses.

predict the trajectory of NPC with fixed state. Though the NPC moves to the same position as the standing NPC, pedestrians are infected by the moving NPC earlier.

The avoidance probability distribution is studied, which is the proportion of the number of pedestrians turning in a zone to the total number of subjects. The calculation formula of the avoidance probability in each zone is shown in Eq. 1.

$$
P_{j}=N_{j} / N_{\text {all }} \quad j \in(1 \ldots 10)
$$

In which, $\mathrm{N}_{j}$ is the number of subjects whose start avoidance points locate in zone $\mathrm{j}$. $\mathrm{N}_{\text {all }}$ represents the total number of subjects. $\mathrm{P}_{j}$ is the avoidance probability in zone $\mathrm{j}$.

In daily life, the pedestrian may become more aggressive and is more willing to avoid the obstacle as she/he is closer to the obstacle (other pedestrians). Therefore, Lv et al. [1] proposed to use the reverse cumulative probability to represent the turning probability. The reverse cumulative probability refers to the ratio of the pedestrians who choose to avoid at the current and previous zones.

In the moving NPC experiments, the pedestrians' turning probability is shown in Fig. 6. The turning probability distribution in the standing NPC experiments is shown in Fig. 6c. The Students $\mathrm{T}$ test is used to compare the differences of the start avoidance points between the two experiments. The results at $30^{\circ}(\mathrm{P}=0.01<0.05), 150^{\circ}(\mathrm{P}=0.04<0.05)$, $180^{\circ}(\mathrm{P}=0.01<0.05)$ intrusion angles have significant differences compared with those in standing NPC experiments. But others at $60^{\circ}(\mathrm{P}=0.12>0.05), 90^{\circ}(\mathrm{P}=0.09>0.05)$, $120^{\circ}(\mathrm{P}=0.28>0.05)$ intrusion angles have no significant difference. These results can help estimate the turning probability of pedestrian at different distance from the CP. In the standing NPC experiments, only 1 person in 37 chose to start avoiding at a position $1.86 \mathrm{~m}$ before the $\mathrm{CP}$.

\subsection{Maximum lateral deviation \& Preference of avoidance direction}

The Maximum Lateral Deviation (MLD), which indicates the maximum deviation from the preset path along $\mathrm{Y}$ axis (perpendicular to the white line), is used to measure the degree of an avoidance. The spatial location of the MLD points are shown in Fig. 7. 

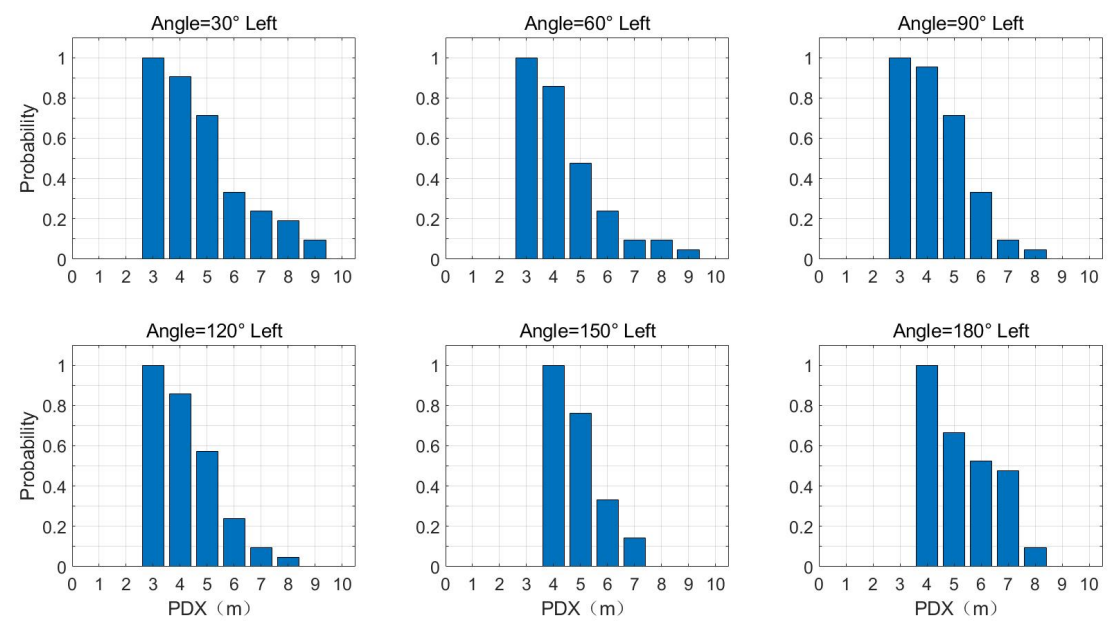

(a) NPC invades from left

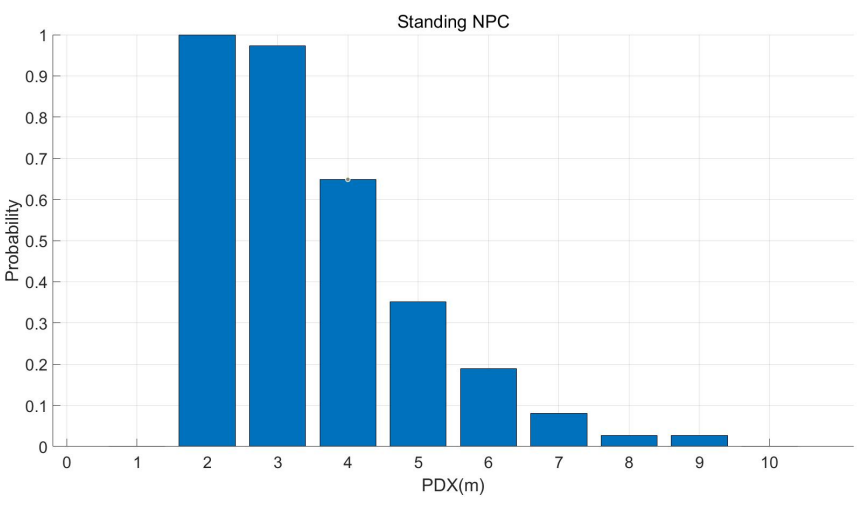

(b) Standing NPC

Figure 6 The reverse cumulative probability of turning. The PDX represents the projection distance between the pedestrian' starting avoidance point and the $\mathrm{CP}$ in the desired direction of movement. 

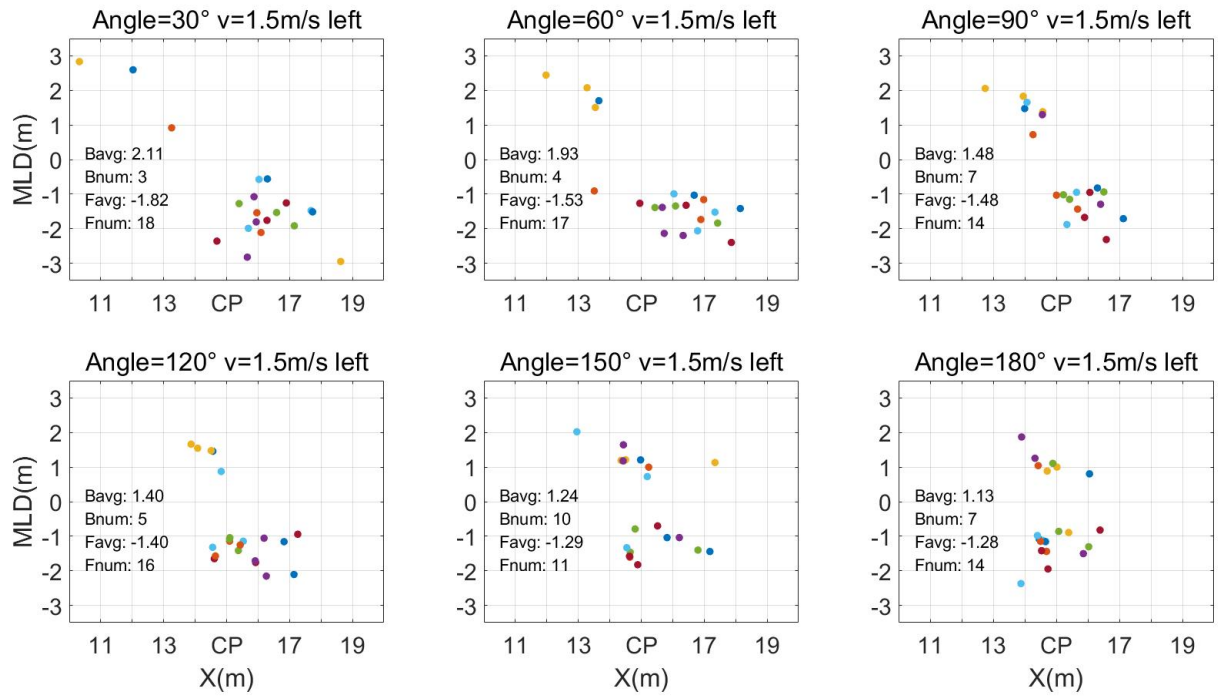

(a) NPC invades from left

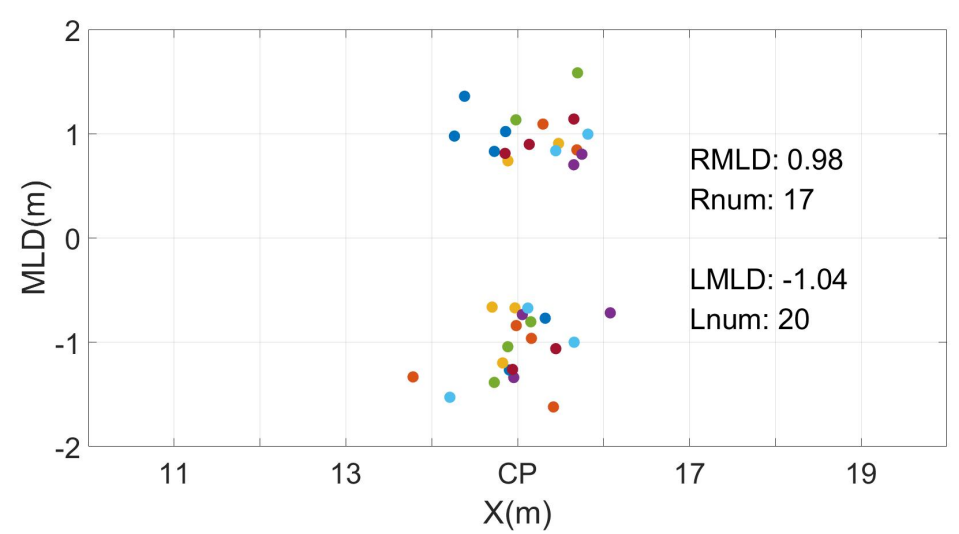

(b) Standing NPC

Figure 7 The MLD position. Bnum: The number of the pedestrians who avoid behind the NPC; Fnum: The number of the pedestrians who avoid in front of the NPC.

In the moving NPC experiments, the avoiding behaviors can be divided into two kinds: avoid in front of the NPC or avoid behind the NPC. Different avoidance behaviors require different MLD values. The average value of MLD under different conditions is listed in Tab. 3. It's found that the average MLD decreases with the increasing intrusion angle of the intruder. The Students T test is used to compare the differences of the MLD between the two experiments. The results at $30^{\circ}(\mathrm{P}=0.00<0.05), 60^{\circ}(\mathrm{P}=0.00<0.05), 90^{\circ}$ $(\mathrm{P}=0.00<0.05), 120^{\circ}(\mathrm{P}=0.00<0.05) 150^{\circ}(\mathrm{P}=0.01<0.05)$ intrusion angles have significant differences compared with those in standing NPC experiments. But results at $180^{\circ}$ $(\mathrm{P}=0.07>0.05)$ intrusion angle have no significant difference.

In addition, the numbers of people in different avoiding directions are recorded in Fig. 7. In order to better compare the avoiding direction preferences, we record the proportion of pedestrians who choose different directions in Tab. 4. It's found that more 


\begin{tabular}{cclll}
\hline \multirow{2}{*}{ Intrusion angle } & \multicolumn{2}{c}{ Moving NPC } & \multicolumn{2}{c}{ Fixed NPC } \\
\cline { 2 - 5 } & Behind & Front & Left & Right \\
\hline $30^{\circ}$ & $2.05(0.63)$ & $1.56(0.45)$ & & \\
$60^{\circ}$ & $2.04(0.57)$ & $1.46(0.55)$ & & \\
$90^{\circ}$ & $1.50(0.56)$ & $1.39(0.68)$ & $1.04(0.31)$ & $0.98(0.23)$ \\
$120^{\circ}$ & $1.32(0.43)$ & $1.32(0.59)$ & & \\
$150^{\circ}$ & $1.16(0.39)$ & $1.27(0.43)$ & & \\
$180^{\circ}$ & $1.12(0.39)$ & $1.17(0.56)$ & & \\
\hline
\end{tabular}

Table 3 The average value of MLD under different conditions (Unit:m). The standard deviation of the data is shown in parentheses.

\begin{tabular}{ccccc}
\hline \multirow{2}{*}{ Intrusion angle } & \multicolumn{2}{c}{ Moving NPC } & \multicolumn{2}{c}{ Fixed NPC } \\
\cline { 2 - 5 } & Behind & Front & Left & Right \\
\hline $30^{\circ}$ & $12 \%$ & $88 \%$ & & \\
$60^{\circ}$ & $17 \%$ & $83 \%$ & & \\
$90^{\circ}$ & $29 \%$ & $71 \%$ & $54 \%$ & $46 \%$ \\
$120^{\circ}$ & $31 \%$ & $69 \%$ & & \\
$150^{\circ}$ & $60 \%$ & $40 \%$ & & \\
$180^{\circ}$ & $57 \%$ & $43 \%$ & & \\
\hline
\end{tabular}

Table 4 Proportion of the pedestrians avoiding in front of and behind the NPC

pedestrians prefer to avoid in front of the NPC at $30^{\circ}, 60^{\circ}$ and $90^{\circ}$ intrusion angles. Because under these conditions, the MLDs required to avoid in front of the NPC are lower than behind NPC. But this preference gradually decreases with the increasing intrusion angle of the intruder and disappears when the angle reaches $180^{\circ}$ (the MLDs are similar in both avoiding side). Pedestrians in the standing NPC experiments behave similarly to those in the moving NPC at $180^{\circ}$ intrusion angle: there is no obvious preference of avoiding side. Because there is no significant difference between the MLDs in left and right side in standing NPC experiments. Low MLD can shorten the pedestrians' avoidance length, reduce their energy consumption, and help them reach the target faster. The difference of MLD in avoiding sides results in the preference of avoiding side.

\section{Conclusion}

The pedestrians' avoidance behaviors under a moving and standing NPC are studied in this paper. According to the trajectories, the avoidance process can be divided into three stages: Judgment stage, Avoidance stage, Return stage. This paper mainly studies the differences of the characteristic points (Start turning, MLD) between moving experiments and standing experiments. The followings are the summary of conclusions.

The transition point of the first stage and the second stage: the starting avoidance point 
is studied. In the moving NPC experiments, the start avoidance point has a strong correlation with the intrusion angle. The average distance between the starting avoidance points and $\mathrm{CP}$ decrease and then increase with the increasing intrusion angle of the intruder. In the standing NPC experiments, the starting avoidance points are closer to the CP than most in the moving NPC experiments. In the standing NPC experiment, due to the stable state of the NPC, pedestrians do not need to predict the trajectory of the NPC, so that the pedestrians will start to avoid in the range closer to the collision point. Then, the starting avoidance probability of pedestrians is further studied in the two experiments, which is in form of the reverse cumulative probability. The distribution of start avoiding points in standing NPC experiments is similar to that at $60^{\circ}, 90^{\circ}$ and $120^{\circ}$ intrusion angles in moving NPC experiments. In addition, the reverse cumulative probability can help to predict the probability of starting avoidance of pedestrian facing one intruder.

The transition point of the second stage and the third stage: the Maximum lateral deviation point is studied. In moving NPC experiments, the MLD decreases with the increasing intrusion angle, whether the NPC invades from left or right side. Under small intrusion angle conditions, the MLD detouring behind the NPC is larger than detouring in front of the NPC. The larger the MLD is, the greater the avoidance costs. The pedestrians need to walk longer distance to avoid the NPC. Therefore, they prefer to avoid potential conflicts by detouring in front of the NPC. But the required MLDs in two sides are similar at $180^{\circ}$ intrusion angle, where the preference disappears. In the standing experiments, the required MLDs in left-hand and right-hand are similar (left: $1.04 \mathrm{~m}$, right: $0.98 \mathrm{~m}$ ), pedestrians have no preference of avoiding side. The probabilities of detouring behind and in front of the NPC in two experiments are obtained, which helps to predict the avoiding side of pedestrians facing one intruder.

Based on VR experiments, the differences in pedestrians' avoidance behaviors facing fixed obstacles and moving obstacles are observed. The differences in results are quantitatively presented.The conclusions of this paper help to establish the obstacle avoidance models and design the moving rules of humanoid robots. But the current research on the pedestrians' avoidance behaviors is limited and incomplete, which need to be further studied. For example, the speed of pedestrian is fixed in this study. However, pedestrians may slow down or stop moving to avoid obstacles in real life. In the future, we will study the difference in avoidance strategy of pedestrian with changing speed when facing fixed and moving obstacles.

Acknowledgements The authors acknowledge the foundation support from the National Natural Science Foundation of China (Grant No.U1933105, 72001095, 71704168), from Anhui Provincial Natural Science Foundation (Grant NO.1808085MG217) and the Fundamental Research Funds for the Central Universities (Grant No.WK2320000040, WK2320000043). 


\section{References}

\section{References}

[1] W. Lv, W.G. Song, J. Ma, Z.M. Fang: A Two-Dimensional Optimal Velocity Model for Unidirectional Pedestrian Flow Based on Pedestrian's Visual Hindrance Field. Ieee Transactions on Intelligent Transportation Systems 14, 1753-1763 (2013), doi:10.1109/tits.2013.2266340

[2] S.M. Bourgaize, B.J. McFadyen, M.E. Cinelli: Collision avoidance behaviours when circumventing people of different sizes in various positions and locations. Journal of Motor Behavior 53, 166-175 (2021), doi:10.1080/00222895.2020.1742083

[3] A.G. Knorr, L. Willacker, J. Hermsdörfer, S. Glasauer, M. Krüger:Influence of person- and situation-specific characteristics on collision avoidance behavior in human locomotion. Journal of Experimental Psychology: Human Perception and Performance 42, 1332-1343 (2016), doi : 10.1037 / xhp0 000223

[4] M. Lappe, M. Huber, Y.-H. Su, M. Krüger, K. Faschian, S. Glasauer, J. Hermsdörfer: Adjustments of Speed and Path when Avoiding Collisions with Another Pedestrian. PLoS ONE 9(2013), doi:10.1371/ journal.pone.0089589

[5] D.R. Parisi, P.A. Negri, L. Bruno: Experimental characterization of collision avoidance in pedestrian dynamics.Physical Review E 94(2016), doi:10.1103/PhysRevE.94.022318

[6] X. Shan, J. Ye, X. Chen: Critical walking space requirement for collision avoidance of pedestrians: an experimental study. In: CICTP 2014: Safe, Smart, and Sustainable Multimodal Transportation Systems, 2369-2380 (2014), doi:10.1061/9780784413623.227

[7] W. Daamen, S. Hoogendoorn, M. Campanella, D. Versluis: Interaction Behavior Between Individual Pedestrians. In:Springer International Publishing, Cham, 1305-1313 (2014), doi:10.1007/978-3-319-02447-9_107

[8] Z. Feng, V.A. González, M. Trotter, M. Spearpoint, J. Thomas, D. Ellis, R. Lovreglio: How people make decisions during earthquakes and post-earthquake evacuation: Using Verbal Protocol Analysis in Immersive Virtual Reality. Safety Science 129(2020), doi:10.1016/j.ssci.2020.104837

[9] M. Kinateder, B. Comunale, W.H. Warren: Exit choice in an emergency evacuation scenario is influenced by exit familiarity and neighbor behavior. Safety Science 106,170-175(2016), doi:10.1016/j.ssci.2018.03.015 
[10] B.A. Baxter, W.H. Warren: Route selection in barrier avoidance.Gait \& Posture 80,192-198(2020), doi:10.1016/j.gaitpost.2020.04.009

[11] J. Bruneau, A.-H. Olivier, J. Pettre: Going through, going around: A study on individual avoidance of groups.Ieee Transactions on Visualization and Computer Graphics 21,520-528(2015), doi:10.1109/tvcg.2015.2391862

[12] S.D. Lynch, R. Kulpa, L.A. Meerhoff, J. Pettre, A. Cretual, A.-H. Olivier,Collision Avoidance Behavior between Walkers: Global and Local Motion Cues.IEEE Transactions on Visualization and Computer Graphics 24, 2078-2088(2018), doi:10.1109/tvcg.2017.2718514

[13] X. Wei, W. Lv, W. Song, X. Li: Survey study and experimental investigation on the local behavior of pedestrian groups.Complexity 20,87-97(2015), doi:10.1002/cplx.21633 